\title{
Ontology for Objective Flight Simulator Fidelity Evaluation
}

\author{
Umut Durak ${ }^{1}$, Artur Schmidt ${ }^{2}$, Thorsten Pawletta ${ }^{2}$ \\ ${ }^{1}$ Institute of Flight Systems, German Aerospace Center (DLR) \\ ${ }^{2}$ Wismar University of Applied Sciences \\ umut.durak@dlr.de
}

The term simulator fidelity has become enormously important in the scope of simulation research, when assessing training efficiency and the transfer of training to real flight. It is defined as the degree to which a flight simulator matches the characteristics of the real aircraft. Objective simulator fidelity provides an engineering standard, by attacking the fidelity problem with comparison of simulator and the actual flight over some quantitative cues. Research flight simulation encompasses some differences from commercial flight simulation. It requires high flexibility and versatility concerning the cockpit layout and visual and motion systems, as well as flight simulation models. It shoud be easy to modify the flight simulation model or other soft- and hardware components of the simulator. By this, there is a need for an automatic test method, in order to determine the fidelity of the most relevant simulator subsystems, since they are often modified during the life cycle of the simulator. The Institute of Flight Systems (FT) at the German Aerospace Center (DLR) has a reconfigurable flight simulator, the Air Vehicle Simulator (AVES), for research of rotorcraft and fixed-wing aircraft. The study reported in this paper targets a model based testing approach designed to tackle the high flexibility requirement of AVES. This paper presents a metamodel for objective flight simulator evaluation. Metamodeling has been carried out in two levels. An Experimental Frame Ontology (EFO) has been developed adopting experimental frames from Discrete Event System Specification (DEVS), and as an upper ontology to specify a formal structure for simulation test. Then in Objective Fidelity Evaluation Ontology (OFEO) that builds upon EFO, domain specific meta-test definitions are captured.

\section{Introduction}

From the late 1920s, when Edward Link built "the Blue Box" [1], flight simulators have been important elements of aviation. Flight simulators became well accepted as training aids by many aircraft operators before the digital era. Highly sophisticated flight simulators have been employed commercially within civil and military flight training organizations in order to enhance pilot skills.

In the 1980s, the aeronautics research community started using flight simulators for developing and experimenting advanced concepts and conducting aviation human factors research. Some of the first examples of research flight simulators include ATTAS Ground Based Simulator from German Aerospace Center (DLR) [2] [3], NASA Crew Vehicle Systems Research Facility in Ames Research Center [4] and Visual Motion Simulation and Cockpit Motion Facility from Langley Research Center [5]. Some more recent examples are the Air Vehicle Simulator (AVES) of DLR [6], HELIFLIGHT from the University of Liverpool [7], NASA Ames Vertical Motion
Simulator [8] and SIMONA of Delft University of Technology [9].

Fidelity in flight simulation can be defined as the degree to which a flight simulator matches the characteristics of the real aircraft. It became more and more important within the scope of flight simulation research as its effect on training efficiency and transfer of training to real flight became better understood [10]. Objective simulator fidelity assessment provides an engineering standard to qualify the degree of fidelity through objective measures. It approaches the fidelity problem with comparison of simulator and the actual flight over some quantitative cues. Requirements for research flight simulators encompas some differences from commercial flight simulators. They require high flexibility and versatility concerning the cockpit layout and visual and motion systems, as well as flight simulation models. They must allow easy modification of the flight simulation model or other soft and hardware components of the simulator. To allow this, there is a need for an automatic test method, to determine the fidelity of most relevant 
simulator subsystems, since they are often modified during the life cycle of the simulator.

Automated testing can be applied through the use of software to control the execution of tests and a comparison of actual outcomes to the predicted ones. Available test data of the aircraft are used as input signals of the simulator and the output signals of the simulator are compared to the measurements to be presented for the evaluator in a smart format. Braun and Galloway [11] reported their automated fidelity test system that compares directly the flight test results and manual execution of flight tests in simulators. Wang et al. [12] [13] presented Automated Test System (ATS) that measure force function, evaluation function and transport delay with its non-intrusive interface with operator station. Jarvis et al. [14] summarizes the efforts on validation of sensory cues, motion cues, vibration and sound cues, visual cues, transport delays and flight dynamics models in flight simulators.

Previous efforts on automated testing for objective flight simulator evaluation utilized fixed test descriptions. DLR intends to adopt a Model Based Testing (MBT) approach to tackle high flexibility requirement of research flight simulators, such as AVES. MBT can be introduced as the idea of automating test case generation from a test model rather than implementing test cases manually [15]. Test modeling is used to specify test cases, and these test models are translated automatically to executable test cases. Metamodeling is employed to capture the domain specific concepts and constraints for building test models [16].

Metamodel is defined as an explicit model of constructs and rules that are used to define a model [17]. Following Gruber [18], definition of ontology is "explicit specification of shared conceptualization". Moreover, metamodels are categorized as ontologies that are used modellers [17].

This paper focusses on the metamodeling step and will present how concepts and constraints of objective flight simulator evaluation are captured in ontology to create a metamodel for test modeling.

Here, the test case can be defined as a sequence of input stimuli that will be fed to the System Under Test (SUT), namely test inputs and the expected behaviour of the system, namely test oracle (Figure 1) [19].

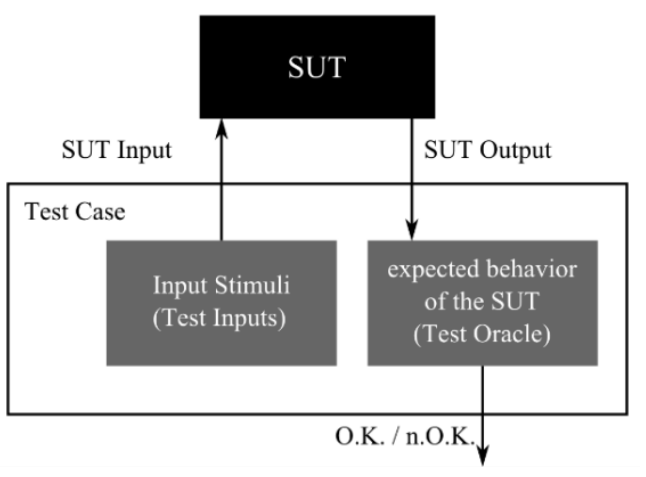

Figure 1 Test Case Structure

Moser et al. [20] stressed that ontologies as machinereadable domain knowledge, which can be utilized for test case generation. Then Nguyen et al. [21] presented a framework for ontology driven test case generation in the context of multi-agent systems. Adopting these ideas, ontologies are employed to structure meta-test definitions. The domain knowledge about the objective validation of simulator systems including the rules for assessing the results of test runs is captured in ontologies.

Zeigler and his colleagues developed the concept of Experimental Frame (EF) [22] [23]. An EF defines the conditions under which a model is to be examined. It comprises of an input generator, a verifier for the desired conditions and an analyzer for the outputs. Following Zeigler et al. [23], the EF is critical for evaluating the model validity. Traoure and Muzy in [24] and Foures et al. in [25] published the usage of the EF approach for specifying invariant validation experiments.

In this research, metamodeling has been carried out on two levels. An EF Ontology (EFO) has been developed as an upper ontology to specify a formal structure for generic simulation test model. Then in Objective Fidelity Evaluation Ontology (OFEO) that builds upon (EFO), domain specific meta test definitions are captured. Protégé [26] is used as the ontology development environment and ontologies are developed using Ontology Web Language (OWL). This paper will present these ontologies after introducing a background on objective fidelity evaluation, experimental frames and ontologies in general.

\section{Background}

\subsection{Objective Fidelity Evaluation}

Fidelity is regarded as a multivariate construct with no consensus among researchers on a single index of 
measurement or definition and it is strongly related to the training task to be performed with the simulator.

There are two approaches to measure simulator fidelity; subjectively and objectively [12]. The subjective approach tries to identify the degree of realism felt by the user. User feedback is structured using rating scales [27]. Although subjective scales are valuable, it is hard to generalize across the scales because the individual opinions and bias of raters [12]. Objective approaches attack the fidelity problem with of simulator and the actual flight over some quantitative cues.

ICAO 9625 Manual of Criteria for the Qualification of Flight Training Devices, $3^{\text {rd }}$ Edition [28] is the well accepted global standard for qualification of flight training devices. The standard specifies seven types of fidelity that correspond to a capability level to provide a certain type of training. For example, simulators classed as 'Type 1' can be used for all training tasks used during completion of Private Pilot License (PPL) training, whereas 'Type 7' is required for some of the training tasks used when awarding 'Type Rating'. Appendix B of the standard specifies the test cases for objective validation of simulators. These test cases include comparison of the results from tests conducted in the simulator and aircraft validation data.

The Royal Aeronautical Society (RAeS) published Aeroplane Simulation Training Device Evaluation Handbook Vol. 1 Objective Testing [29] to ease the implementation and enhance the understanding of objective tests introduced in ICAO 9625. It provides further discussions about the implementation of each test and introduces some example cases with some plots.

ICAO 9625 provides tables that specify each test case with parameters, tolerances and flight conditions. Table 1 shows an example test specification from the standard, for testing the minimum radius.

\begin{tabular}{|l|l|l|l|l|l|l|l|l|}
\hline Test & Tolerance & \multicolumn{6}{|l|}{ Type } \\
\cline { 3 - 8 } & & $\mathbf{1}$ & $\mathbf{2}$ & $\mathbf{3}$ & $\mathbf{4}$ & $\mathbf{5}$ & $\mathbf{6}$ & $\mathbf{7}$ \\
\hline $\begin{array}{l}\text { Minimum } \\
\text { radius turn }\end{array}$ & $\begin{array}{l} \pm 0,9 \mathrm{~m}(3 \mathrm{ft}) \text { or } \pm 20 \% \text { of } \\
\text { aeroplane turn radius }\end{array}$ & & & & & $\checkmark$ & & $\checkmark$ \\
\hline
\end{tabular}

Table 1 Sample Test Specification from ICAO 9625 [28]

Tests are grouped under performance, handling qualities, motion system, visual system and sound system. Among these tests, those under performance and handling qualities headings are related to flight dynamics models, and have no other subsystem or device depandencies. For this reason, they are considered to better suit automation. Therefore, as a first step, this addresses these groups.

The RAeS introduces the benefits of employing automatic testing in objective fidelity evaluation as repeatability, ease and rapidity of conducting tests. The RAeS handbook [29] specifies the features of an automatic testing system as initializing the simulator with the test initial conditions, trimming the aircraft, creating the stimulus if required, using flight controls and finally checking the simulator output against test criteria.

\subsection{Experimental Frame Approach}

The EF approach was originally introduced by Zeigler in [22] in context with the Discrete Event System Specification (DEVS). The objective is the explicit separation between the model and the experiment. Moreover, an EF specifies a limited set of circumstances under witch a model is to be observed. Nowadays, the EF approach belongs to the state of the art and it is used in many modelling and simulation projects including validation experiments [24] [25] [30] [31]. Following Zeigler [22], the formal specification of the EF is given by the 7-tuple:

$$
\mathrm{EF}=\langle\mathrm{T}, \mathrm{I}, \mathrm{O}, \mathrm{C}, \Omega \mathrm{i}, \Omega \mathrm{c}, \mathrm{SU}>
$$

where:

$\mathrm{T}$ is the time base

I is the set of input variables

$\mathrm{O}$ is the set of output variables

$\mathrm{C}$ is the set of control variables

$\Omega i$ is the set of admissible input segments

$\Omega \mathrm{c}$ is the set of admissible control segment

$\mathrm{SU}$ is a set of summary mappings

The EF can be implemented in various ways. Zeigler [22] recommends implementing the EF as a coupled system consisting of a generator, acceptor and a transducer that is conected to a SUT. In our context, the SUT is always a model. For this reason, it is called Model Under Test (MUT). Figure 2 illustrates such a realization of EF coupled to a MUT schematically.

Test inputs are produced by a generator. They have to be admissible input segments of MUT and influence its behavior. The acceptor and transducer form the 
test oracle. Based on output variables, the transducer calculates outcome measures in the form of performance indices, comparative values, statistics etc.

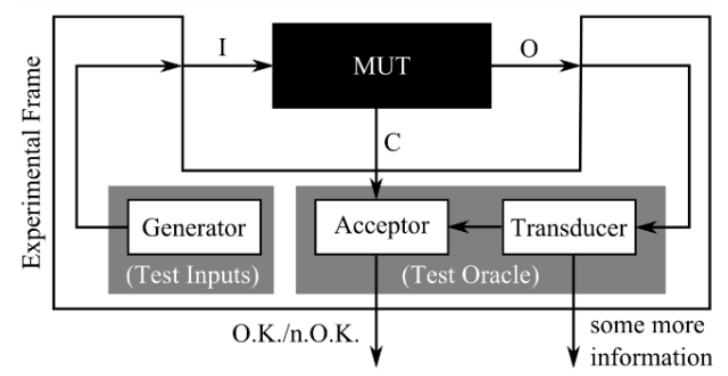

Figure 2 Illustration of EF with MUT.

The acceptor corresponds to a decision unit that decides if an experiment is valid or not. For this purpose the acceptor monitors its inputs and maps them to a specified admissible control segment. In case of violation of the admissible control segment the experiment will not accepted. Beside control variables, the input of an acceptor can be output variables or outcome measures.

The EF approach defines a uniform structure for a systematic experiment specification. The specification has to be coded in the description of an EF. This means that each kind of experiment needs the definition of a distinct EF.

\subsection{Ontologies}

Knowledge in a domain is formalized using concepts, relations, functions, axioms and instances in an ontology. Concepts can be anything about which something is said, and therefore, can be a description of a task, function, action, strategy etc. Taxonomies are widely used to organize the ontological knowledge in domain using generalization/specialization relationship through simple/multiple inheritance. Relationships represent a type of interaction between the concepts of the domain and functions can be regarded as a special kind of relation. Axioms on the other hand are used to model sentences that are always true. They are added to ontology for several purposes, such as constraining the information contained in the ontology, verifying its correctness or deducting new information. Instances are the terms that are used to represent the elements of the domain. They actually represent the elements of the concepts [32].

Ontologies in engineering domain have been developed for various purposes including specifying engineering information systems, integration of engineering applications, supporting engineering design and development. The first efforts on developing engineering ontologies were in the 1990's. The 'PhysSys' [33] was one of the first engineering ontologies based upon system dynamics theory that is practiced in engineering modeling, simulation and design. The PhysSys was developed to formally define how design engineers or the end users of Computer Aided Engineering (CAE) systems understand their domain and to provide a foundation for the conceptual schema for data structuring in engineering databases, libraries and other CAE information systems [33] [34]. The ideas formalized in PhysSys provided a base for the development of a library of reusable models for engineering and design. Fishwick and Miller in [35] discussed the venues of ontology use in modeling and simulation. One of the late examples of ontology use in modeling and simulation is reported by Durak et al. [36] [37]. The group enabled simulation reuse over an ontology driven methodology. Another ontology-based modeling and simulation approach was established by Zeigler with the System Entity Structure and Model Base (SES/MB) framework [22] [23] [38] [39]. Today the SES is an ontology framework for conceptual system modeling and for specification of a set of modular hierarchical system structures.

\section{Experimental Frame Ontology}

The EFO forms the upper level of the metamodel for objective flight simulation evaluation. The previously introduced EF approach is used to specify a formal structure of generic test cases. Hence, every test case has to be specified according to the EF definition in Section 2.2.

Figure 3 illustrates the entity hierarchy of the EFO in Protégé. The first layer consists of three entities: Computational Unit, Informational Unit and the EF. Computational Units comprises the generic Acceptor, Transducer and Generator which will be presented as executable blocks in a test case. The Information Unit defines basic entities of an EF. For practical usage, there are some differences to the theoretical definition of an EF according to the 7-tuple. The Experimental Frame entity conforms to the actual EF.

Futhermore particular properties are implemented to define the relations between the entities. For example the properties composed $O f$ and definedBy makes clear 
that any EF is a composition of Computational Unit and is defined by the Informational Unit.

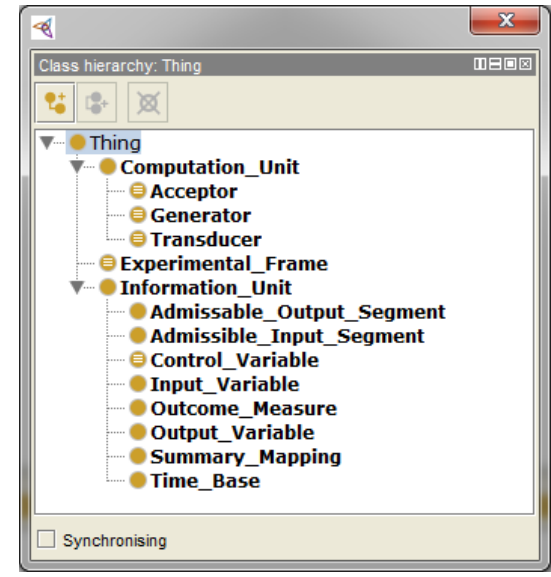

Figure 3 Entity Hierarchy of the Experimental Frame Ontology

As a result we obtain a generic EF which conforms to a generic test case. Thus, any test case will have the unique structure as shown in Figure 4 on its top level.

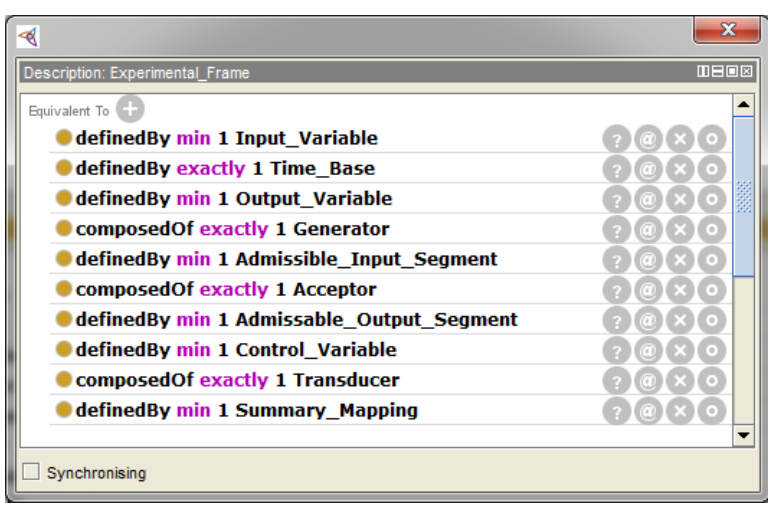

Figure 4 Description of a Generic Experimental Frame

The EFO forms the basis fot the OFEO that will define test cases in detail.

\section{Objective Fidelity Evaluation Ontology}

OFEO is constructed by extending the upper level EFO that specifies any test case that will be applied to MUT using experimental frames formalism. The hierarchy of OFEO using Protégé is depicted in Figure 5. The elements from EFO can be traced in this hierarchy.

Each objective validation test case described in ICAO 9625 under performance and handling qualities are specified by an experimental frame. Thus, each test possesses a Generator, Transducer and an Acceptor. The specification of these three entities will inherently describe how this specific test will be exercised.
These three entities will constitute the automatic test system.

Following the features of automated test systems introduced in the RAeS Handbook [29], the Generator is described as the component to initialize the test with initial conditions and trim the aircraft and create the stimulus following the ones from the flight test using the flight controls. Hence, the Generator is interpreted as test independent. On the other hand, the Transducer is described as the component that will compute Outcome Measures that are required for the Acceptor for a specific test.

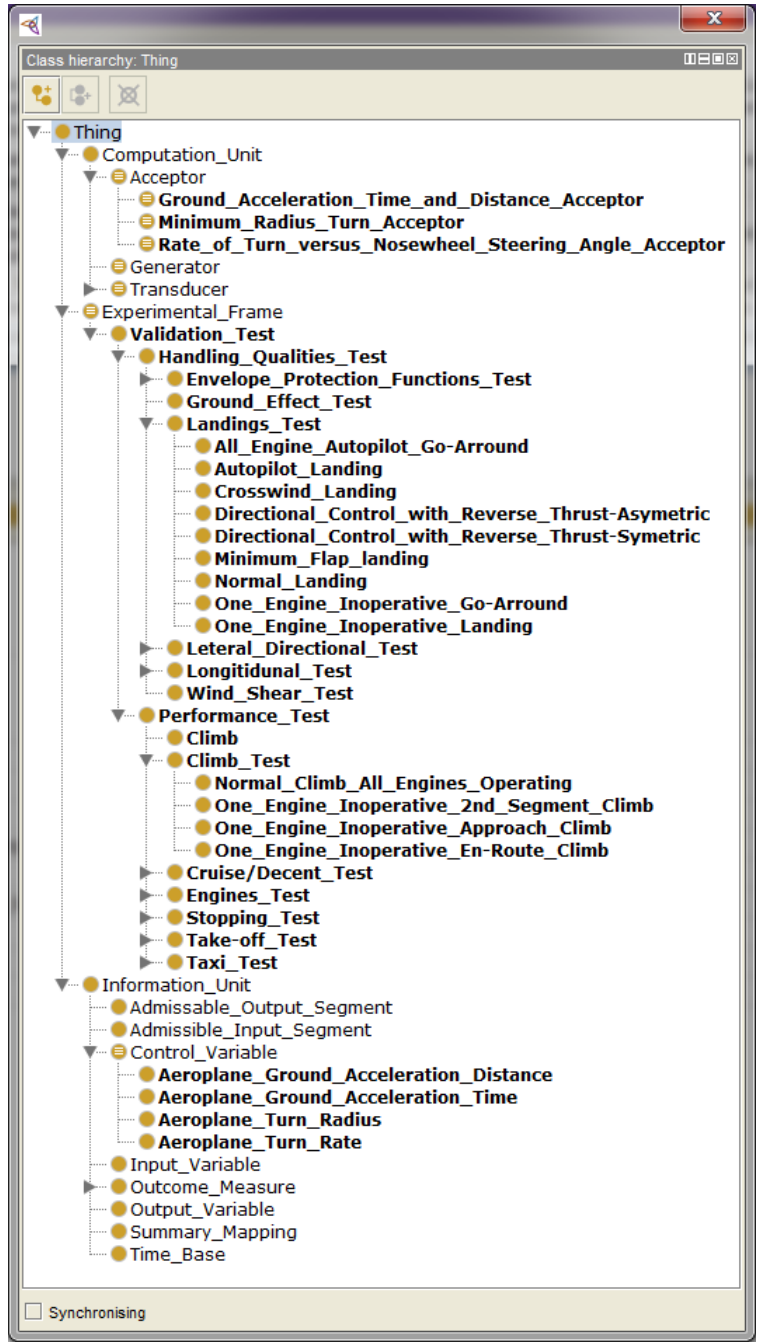

Figure 5 Objective Fidelity Evaluation Ontology Hierarchy

As an example, the Minimum Turn Radius test requires a Simulated Turn Radius to be computed from a simulation output. Or likewise, Rate of Turn versus Nosewheel Steering Angle test requires Simulated Turn Rate value to be computed. So, a specific trans- 
ducer is defined for every test. Lastly, the Acceptor is described as the component that checking the MUT against test criteria. Since every test has a particular criterion, an Acceptor is defined for each test. Accordingly, we are expecting to have particular Control Variables for each test.

\begin{tabular}{||l|l|l||}
\hline Description: Minimum_Radius_Turn & \\
\hline Equivalent To + \\
OdefinedBy some Simulated_Turn_Radius
\end{tabular}

Figure 6 Minimum Radius Turn Test Description

Figure 6 presents an example test description in Protégé. The Minimum Turn Radius Test is specified with a specific Acceptor, Transducer and Control Variables, Simulated Turn Radius and Aeroplane Turn Radius. On the other hand, it inherits the properties of an experimental frame. So it will also have a Generator, Input Variables, Output Variables, Admissible Input Segments, Admissible Output Segments and a Summary Mapping. It is clear that input and output variables of the flight simulator are application specific but does not vary with test cases, so generic definitions are kept for these variables and admissible segments.

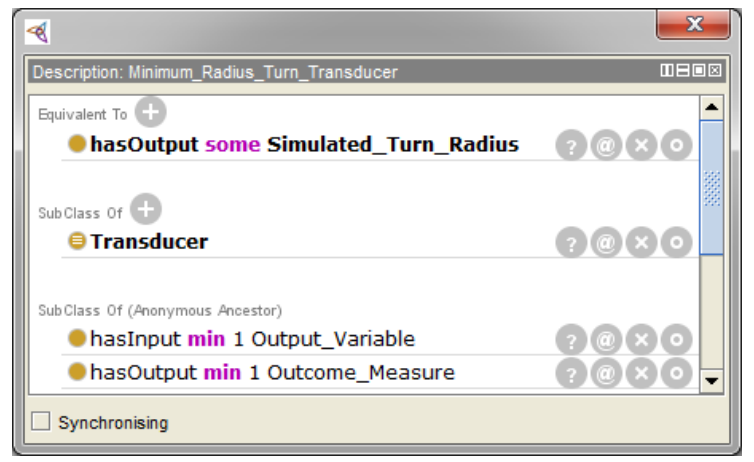

Figure 7 Minimum Radius Turn Transducer Description
Minimum Radius Turn Transducer (Figure 7) is defined with an output Simulated Turn Radius while it also inherits the properties of a Transducer. It will be using Output Variables for computing the outcome measure. Since the computation of the outcome measure is largely implementation specific, ontology does not have any knowledge about it.

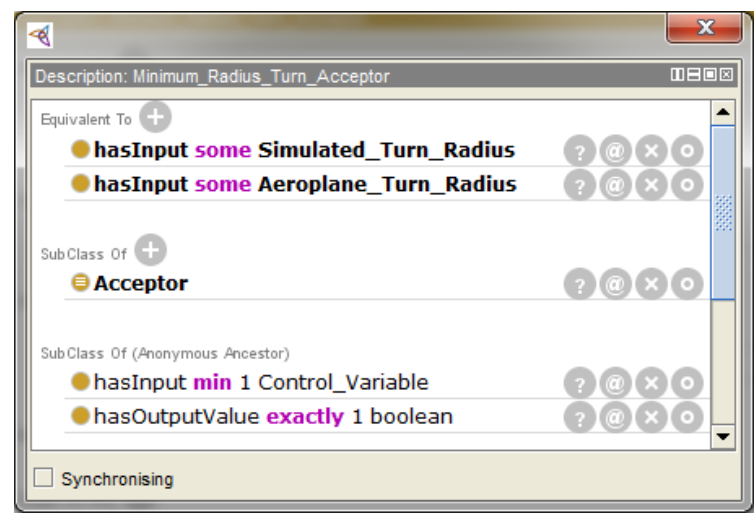

Figure 8 Minimum Radius Turn Acceptor Description

As an example, the Minimum Radius Turn Acceptor is depicted in Figure 8. Since each of the tests have distinct criteria, the acceptors will have particular inputs. Accordingly, Minimum Radius Turn Acceptor is described with Simulated Turn Radius and Aeroplane Turn Radius inputs. On the other hand the output of the acceptor is always a Boolean if the criterion is matched or not.

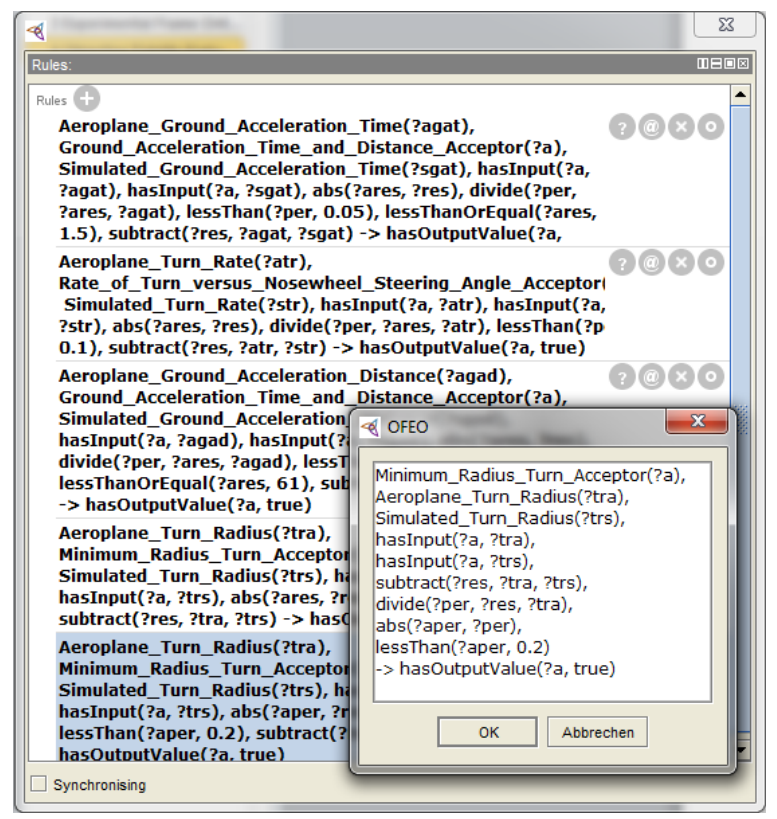

Figure 9 Rules for Acceptors 
Semantic Web Rule Language (SWRL) [40] is used to formalize the acceptance criteria. The rules specify how the inputs of the acceptor are used to compute if the test is successful or not. In Figure 9, the rule in the front windows says that Minimum Radius Turn Acceptor has a true output when the difference between the simulated and the real minimum turn radius is smaller than $20 \%$.

\section{Conclusion}

This paper presents an ontology based metamodeling approach for objective flight simulator evaluation. EFO adopts the concept of EF from DEVS, as an upper ontology to specify a formal structure for test cases. Thus with EF, concepts of MBT could be formally specified. This established a solid base for modeling specific test cases. Then in OFEO that builds upon EFO, domain specific meta-test definitions are modeled. While OWL is used as the ontology language; SWRL is employed to capture the rules. Protégé is utilized as the ontology development environment.

This effort assembled the first step of developing an automatic test system for simulator fidelity evaluation. The next step is to design and establish the computational infrastructures that will interprete the knowledge captured in these ontologies and execute the tests.

\section{References}

[1] D. Allerton. Principles of Flight Simulation. John Wiley \& Sons, Ltd, West Sussex, United Kingdom, 2009.

[2] P. Saager. Real-Time Hardware-in-the-Loop Simulation for 'ATTAS' and 'ATTHeS' Advanced Technology Flight Test Vehicles. in AGARD Guidance and Control Panel, 50th Symposium, Izmir, Turkey, 1990.

[3] S. Klaes. ATTAS Ground Based System Simulator -An Update-. In: AIAA Modeling and Simulation Technologies Conference and Exhibit, Denver, CO, 2000.

[4] B. Sullivan and P. Soukup. The NASA 747-400 Flight Simulator: A Natonal Reseource fir Aviation Safety Research. In: AIAA Flight Simulation Technologies Conference, San Diego, CA, 1996.

[5] R. Smith. A Description of the Cockpit Motion Facility and the Research Flight Deck Simulator.
In: AIAA Modeling and Simulation Technologies Conference and Exhibit, Denver, CO, 2000.

[6] H. Duda, T. Gerlach, S. Advani and M. Potter. Design of the DLR AVES Research Flight Simulator. In: AIAA Modeling and Simulation Technologies (MS) Conference, Boston, MA, 2013.

[7] M. White and G. Padfield. The Use of Flight Simulation for Research and Teaching in Acedemia. In: AIAA Atmospheric Flight Mechanics Conference and Exhibit, Keystone, CO, 2006.

[8] S. Advani, D. Giovannetti and M. Blum. Design of a Hexapod Motion Cueing System for NASA Ames Vertical Motion Simulator. In: AIAA Modeling and Simulation Technologies Conference and Exhibit, Monterey, California, 2002.

[9] O. Stroosma, R. van Paassen and M. Mulder. Using the Simona Research Simulator for Human-Machine Interaction Research. In: AIAA Modeling and Simulation Technologies Conference and Exhibit, Austin, Texas, 2003.

[10] T. Longride, J. Bürki-Cohen, T. Go and A. Kendra. Simulator Fidelity Considerations for Training and Evaluation of Today's Airline Pilots. In: Proceedings of the 11th International Symposium on Aviation Psychology, Columbus, $\mathrm{OH}, 2001$

[11] D. Braun and R. Galloway, Universal Automated Flight Simulator Fidelity Test System. In: AIAA Modeling and Simulation Technologies Conference and Exhibit, Rhode Island, 2004.

[12] C. Wang, J. He, G. Li and J. Han. An Automated Test System for Flight Simulator Fidelity Evaluation. Journal of Computers, vol. 4(11), 2009.

[13] C. Wang, J. Han, G. Li and H. Jiang. Flight Simulator Fidelity Evaluation Automated Test System Analysis. In: 2008 International Workshop on Education Technology and Training, Shanghai, China, 2008.

[14] P. Jarvis, D. Spira and B. Lalonde. Flight Simulator Modeling and Validation Approaches and Pilot-in-the-loop Fidelity. In: AIAA Modeling and Simulation Technologies Conference and Exhibit, Honolulu, Hawaii, 2008.

[15] J. Zander, I. Schieferdecker and P. Mosterman, A Taxononomy of Model-Based Testing for Embedded Systems from Multipke Industry Domains. In: Model-Based Testing for 
Embedded Systems, Boca Rato, CRC Press, 2012, pp. 3-23.

[16] A. Guduvan, H. Waselynck, V. Wiels, G. Durrieu, Y. Fusero and M. Schieber. A MetaModel for Tests of Avionics Embedded Systems. In: Modelsward, Barcelona, Spain, 2013.

[17] D. Gasevic, D. Djuric and V. Devedzic. Model Driven Architecture and Ontology Development. Springer-Verlag, Berlin, 2006.

[18] T. Gruber. Toward Principles for the Design of Ontologies Used for Knowledge Sharing. Int. Journal of Human-Computer Studies, vol. 43, pp. 907-928, 1995.

[19] S. Weissleder. Test Models and Coverage Criteria forAutomatic Model-Based Test Generation with UML State Machines. Humboldt-Universität zu Berlin, Berlin, 2010.

[20] T. Moser, G. Düee and S. Biffl, Ontology-Based Test Case Generation For Simulating Complex Production Automation Systems. In: SEKE 2010, San Fransisco Bay, USA, 2010.

[21] C. Nguyen, A. Perini and P. Tonella. Ontologybased Test Generation for Multiagent Systems. In: 7th International Joint Conference on Autonomous Agents and Multiagent Systems, Estoril, Portugal, 2008.

[22] B. Zeigler. Multifacetted Modelling and Discrete Event Simulation. Academic Press Professional, Inc., 1984.

[23] B. Zeigler, H. Praehofer and T. Kim. Theory of Modeling and Simulation: Integrating discrete event and continuous complex dynamic systems. Academic Press, Inc., 2000.

[24] M. Traoré and A. Muzy. Capturing the dual relationship between simulation models and their context. Simulation Modelling Practice and Theory, 14, pp. 126-142, 2006.

[25] D. Foures, V. Albert and A. Nketsa. Simulation Validation Using the Compatibility between Simulation Model and Experimental Frame. In: 45th Summer Simulation Multi-conference, Toronto, Canada, 2013.

[26] K. Holger, M. Horridge, M. Musen, A. Rector, R. Stevans, N. Drummond, P. Lord, N. Noy, J. Seidenberg and $\mathrm{H}$. Wangl. The Protege $O W L$ Experience. In: OWLED, Galway, Ireland, 2005.

[27] P. Perfect, E. Timson, M. White, R. Erdos, A. Gubbels and A. Berryman. A Rating Scale for Subjective Assesment of Simulator Fidelity. In: 37th European Rotorcraft Forum, Gallarate, Italy, 2011.

[28] ICAO, Manual Criteria for the Qualification of Flight Training Devices. ICAO, Quebec,
Canada, 2009.

[29] RAeS, Aeroplane Flight Simulation Training Device Evaluation Handbook Vol.1 Objective Testing. RAeS, London, 2009.

[30] B. Nader and J. B. Filippi, An Experimental Frame for the Simulation of Forest Fire Spread. In: Proceeding of the 2011 Winter Simulation Conference, Phoenix, Arizona, USA, 2011.

[31] A. Zengin and M. Ozturk, Formal verification and validation with DEVS-Suite: OSPF Case study. Simulation Modelling Practice, vol. 29, pp. 193-206, 2012.

[32] O. Corcho and A. Perez, Evaluating Knowledge Representation and Reasoning Capabilities of Ontology Specification Languages. In: ECAI'00 Workshop on Applications of Ontologies and Problem Solving Methods, Berlin, Germany, 2000.

[33] W. Borst, J. Akkermans, A. Pos and J. Top. The PhysSys Ontology for Physical System. In: QR'95 Ninth International Workshop on Qualitative Reasoning, Amsterdam, Netherlands, 1995.

[34] W. Borst and J. Akkermans. Engineering Ontologies. International Journal of HumanComputer Studies, vol. 46 (2/3), pp. 365-406, 1997.

[35] P. Fishwick and J. Miller. Ontologies for Modeling and Simulation: Issues and Approaches. In: Winter Simulation Conference, Washington, DC, 2004.

[36] U. Durak, H. Oguztuzun and K. Ider. Ontology Based Trajectory Simulation Framework. Journal of Computing and Information Science in Engineering, vol. 8(1), March 2008.

[37] U. Durak, H. Oguztuzun and K. Ider. Ontology Based Domain Engineering for Trajectory Simulation Reuse. Journal of Software Engineering and Knowledge Engineering, vol. 19(8), December 2009.

[38] B. Zeigler. Modeling \& Simulation-Based Data Engineering: Introducing pragmatics into ontologies for net-centric information exchange. Academic Press, Inc., 2007.

[39] B. Zeigler and H. Sarjoughian. Guide to Modeling and Simulation of Systems of Systems. Springer, 2013.

[40] I. Harrocks, P. Patel-Schneider, H. Boley, S. Tabet, B. Grosof and M. Dean. SWRL: $A$ Semantic Web Rule Language Combining OWL and RuleML. W3C, Canada, 2004. 